\title{
PRIVILEGED REGIONS IN CRITICAL STRIPS OF NON-LATTICE DIRICHLET POLYNOMIALS
}

\author{
G. MORA AND J.M. SEPULCRE
}

\begin{abstract}
This paper shows, by means of Kronecker's theorem, the existence of infinitely many privileged regions called $r$-rectangles (rectangles with two semicircles of small radius $r$ ) in the critical strip of each function $L_{n}(z):=$ $1-\sum_{k=2}^{n} k^{z}, n \geq 2$, containing exactly $\left[\frac{T \log n}{2 \pi}\right]+1$ zeros of $L_{n}(z)$, where $T$ is the height of the $r$-rectangle and [.] represents the integer part.
\end{abstract}

\section{INTRODUCTION}

The study of the zeros of exponential polynomials is a topic which appears in the first third of the twentieth century in relation with the development of differential equation theory. At this point we quote Wilder's paper [13], firstly, because it constitutes a good example about the mentioned relation of the zeros with differential equations and, secondly, because in that paper we can find one of the first formulae to determine the number of zeros of an exponential sum of the form

$$
f(z):=1+Q_{0} e^{z}+Q_{1} e^{c_{1} z}+Q_{2} e^{c_{2} z}+\ldots+Q_{k-2} e^{c_{k-2} z},
$$

inside a rectangle of the critical strip where its zeros are located. Indeed, by assuming the $Q$ 's are complex constants, and in particular $Q_{k-2}$ and at least one other are different from zero, and the $c$ 's are real constants (called frequencies) such that

$$
1<c_{1}<c_{2}<\ldots<c_{k-2}
$$

Wilder proved that in a rectangle $R$ of length $l$ of the critical strip of $f(z)$, the number of roots $N(R)$ satisfies

$$
\left|N(R)-\frac{c_{k-2}}{2 \pi} l\right| \leq k-1
$$

Along the line of Wilder's paper [13] are the works of Tamarkin [10, 11] about the zeros of certain functions "in connexion with some general problems of the expansion theory for linear differential equations" (the words in inverted commas belong to Tamarkin [10, p. 66]). Analogous results we find in Turan's article [12], where he attributes to Polya [9] the following theorem:

$$
\text { "If } z=x+i y
$$

$$
\mu_{1}<\mu_{2}<\ldots<\mu_{l}
$$

and $P_{\nu}(z)$ is for $\nu=1,2, \ldots, l$ a polynomial of degree $\leq m_{\nu}-1$ with

$$
\begin{gathered}
m_{1}+m_{2}+\ldots+m_{l}=n \\
P_{1}(z) P_{l}(z) \neq 0,
\end{gathered}
$$

${ }^{1}$ Preprint submitted to Complex Analysis and Operator Theory. April, 2012.

2000 Mathematics Subject Classification. Primary: 30Axx; 30D05. Secondary: 11J99; 65Axx.

Key words and phrases. Zeros of exponential polynomials; non-lattice Dirichlet polynomials; Kronecker theorem. 
then the number $N\left(g_{l}, a, a+d\right)$ of the zeros (according to multiplicity) of the function

$$
g_{l}(z)=\sum_{\nu=1}^{l} P_{\nu}(z) e^{i \mu_{\nu} z}
$$

in the infinite vertical strip

$$
a \leq x \leq a+d
$$

satisfies the inequality

$$
\left|N\left(g_{l}, a, a+d\right)-\frac{\left(\mu_{l}-\mu_{1}\right) d}{2 \pi}\right| \leq n-1 . ”
$$

In [4], Langer showed that the number of zeros $N(R)$ of the exponential sum

$$
\Phi(z)=\sum_{j=0}^{n} a_{j} e^{c_{j} z}, a_{j} \in \mathbb{C} \backslash\{0\} ; 0=c_{0}<c_{1}<\ldots<c_{n}
$$

within a rectangle $R$ cut from the critical strip by two arbitrary lines $y=y_{1}$ and $y=y_{2}$, chosen so that on neither of them there lies a zero of $\Phi(z)$, verifies

$$
\left|N(R)-\frac{c_{n}}{2 \pi}\left(y_{2}-y_{1}\right)\right| \leq n
$$

Recently, for the partial sums

$$
F_{X}(s)=\sum_{n \leq X} n^{-s}
$$

with $s=\sigma+i t$ a complex variable and $X$ a real number greater than or equal to 2, Gonek and Ledoan proposed in [2, Theorem 2] the formula

$$
\left|N_{X}(T)-\frac{T}{2 \pi} \log [X]\right|<\frac{X}{2},
$$

which determines the number of zeros of $F_{X}(s), N_{X}(T)$, for the special case that these zeros to have ordinates in the interval $[0, T]$, where $[X]$ denotes the integer part of $X$.

The generalization of the foregoing formulae to the most large class of almostperiodic functions can be found in Levin's book [8].

As we can see in the extensive literature about the topic of the zeros of an exponential polynomial, the formulae to determine the number of its zeros in an arbitrary rectangle $R$ of the critical strip where they are situated, have a common thing: all them contain a principal term given by the greatest frequency of the function multiplied by the height of the rectangle and divided by $2 \pi$, and a bound which expresses the maximum error with respect to the exact number of those zeros inside $R$. Indeed, all formulae (1.1)-(1.4) contain the same principal term and point out that the error is of the same order that the degree of the exponential sum, with the exception of (1.4), where the error reduces to one half because that formula applies to a special rectangle cut from the critical strip by the lines $y=0$ and $y=T$, and then as the function $F_{X}(s)$ is strictly positive on $y=0$, the contribution to the variation of the argument of $F_{X}(s)$ along the bottom of $R$ is zero.

The functions

$$
L_{n}(z):=1-\sum_{k=2}^{n} k^{z}, n>2
$$


are the prototype of non-lattice Dirichlet polynomials, that is, functions of the form

$$
1-\sum_{k=2}^{n} m_{k} a_{k}^{z} ; m_{k} \in \mathbb{C}, a_{k}>0,
$$

for which the group $G:=\mathbb{Z} \log a_{2}+\ldots+\mathbb{Z} \log a_{n}$ is dense in $\mathbb{R}$. The importance of the zeros of the $L_{n}(z)$ 's is due to the connection between them and the fractal string concept, introduced by Lapidus and Pomerance in [5]. In fact, the zeros of $L_{n}(z)$ are the poles of the meromorphic continuation of the geometric zeta function, $\zeta_{\mathcal{L}}(z):=\frac{1}{L_{n}(z)}$ (see [7, p.97] and especially Lapidus and van Frankenhuijsen's book [6, Chapters 2-3] where we can find a complete discussion and stronger results on the zeros of Dirichlet polynomials).

Our purpose in this paper is to point out that a reiterated application of Kronecker's theorem [3, Theorem 444] allows us to prove the existence of infinitely many special regions, the mentioned $r$-rectangles $R_{n T}$, in the critical strip of every function $L_{n}(z), n \geq 2$, where we can exactly count the number of its zeros inside them by the formula

$$
N_{n}(T)=\left[\frac{T \log n}{2 \pi}\right]+1
$$

where $T$ is the height of $R_{n T}$ and [.] denotes the integer part. That means that for these $r$-rectangles our formula (1.5) keeps the common principal term of all preceding formulae (1.1)-(1.4) but the error has been reduced to zero.

\section{The Formula}

For every integer $n \geq 2$

$$
L_{n}(z):=1-2^{z}-\ldots-n^{z}
$$

is an entire function of order 1 , exponential type $\ln n$, and it has infinitely many zeros not all them situated on the imaginary axis, except $L_{2}(z)$, whose zeros $z_{k}$ are explicitly given by the formula

$$
z_{k}=\frac{2 k \pi i}{\ln 2}, k \in \mathbb{Z}
$$

On the other hand, since for any $y$

$$
\lim _{x \rightarrow-\infty} L_{n}(x+i y)=1
$$

and

$$
\lim _{x \rightarrow+\infty} \frac{L_{n}(x+i y)}{-n^{x+i y}}=1,
$$

there exist two values of $x, x_{n_{1}}<0<x_{n_{2}}$, such that

$$
\left|L_{n}(z)-1\right|<1 \text { for all } z \text { with } \operatorname{Re} z \leq x_{n_{1}}
$$

and

$$
\left|\frac{L_{n}(z)}{n^{z}}+1\right|<1 \text { for all } z \text { with } \operatorname{Re} z \geq x_{n_{2}} .
$$

Therefore, each function $L_{n}(z)$ has all its zeros comprised in a vertical strip $S_{n}$, called critical strip, defined by

$$
S_{n}:=\left\{z=x+i y: a_{n} \leq x \leq b_{n}\right\},
$$


whose bounds

and

$$
a_{n}:=\inf \left\{\operatorname{Re} z: L_{n}(z)=0\right\}
$$

$$
b_{n}:=\sup \left\{\operatorname{Re} z: L_{n}(z)=0\right\},
$$

because (2.4) and (2.5), are both finite. On the other hand, (2.4) and (2.5) also imply that

$$
0<L_{n}(x) \text { for all } x \leq x_{n_{1}}
$$

and

$$
0>L_{n}(x) \text { for all } x \geq x_{n_{2}},
$$

and then $L_{n}(z)$ has a real root, say $\alpha_{n}$, which is unique noticing that the derivative $L_{n}^{\prime}(x)<0$ for all $x \in \mathbb{R}$. Thus, as in particular at the point $\alpha_{n}$ one has $L_{n}^{\prime}\left(\alpha_{n}\right) \neq 0$, by applying [1, Theorem 3.3.3], there exists a sufficiently small real number $r_{n}$ such that

for which the properties

$$
\frac{\pi}{\log n}>r_{n}>0
$$

i) $L_{n}^{\prime}(z) \neq 0$ for all $\left|z-\alpha_{n}\right| \leq r_{n}$,

ii) $L_{n}(z) \neq 0$ for all $0<\left|z-\alpha_{n}\right| \leq r_{n}$

iii) $L_{n}(z)$ is one-to-one on $\left|z-\alpha_{n}\right| \leq r_{n}$,

hold.

The $r_{n}$ 's are crucial to define the $r$-rectangles.

Definition 1. For each integer $n \geq 2$, given the previous $r_{n}$, an $r_{n}$-rectangle, denoted by $R_{n, T}$, is a region whose boundary is defined by the vertical lines $x=a_{n}^{\prime}$, $x=b_{n}^{\prime}$ and the paths

$$
\begin{gathered}
\Gamma_{n}^{\prime}:=\left\{(x, y): a_{n}^{\prime} \leq x \leq \alpha_{n}-r_{n} ; y=0\right\} \cup\left\{z:\left|z-\alpha_{n}\right|=r_{n} ; \operatorname{Im} z<0\right\} \cup \\
\cup\left\{(x, y): \alpha_{n}+r_{n} \leq x \leq b_{n}^{\prime} ; y=0\right\}
\end{gathered}
$$

and

$$
\Gamma_{n, T}^{\prime}:=\left\{z+i T: z \in \Gamma_{n}^{\prime}\right\}
$$

where $a_{n}^{\prime}$ and $b_{n}^{\prime}$ are arbitrary real numbers verifying $a_{n}^{\prime}<a_{n}, b_{n}^{\prime}>b_{n}$ and $T$ is a positive number such that $L_{n}(z)$ has no zero on $\Gamma_{n, T}^{\prime}$.

Remark 2. The values of $T$ for which formula (1.5) will be valid will be specified in the proof of the next theorem. We note that the $r_{n}$-rectangles are counterclockwise oriented.

Theorem 3. In the critical strip of each $L_{n}(z):=1-2^{z}-\ldots-n^{z}, n \geq 2$, there exist infinitely many $r_{n}$-rectangles $R_{n, T}$ such that the number of its zeros, $N_{n}(T)$, inside each $R_{n, T}$ is given by the formula

$$
N_{n}(T)=\left[\frac{T \log n}{2 \pi}\right]+1,
$$

where $T$ is the height of $R_{n, T}$ and [.] denotes the integer part.

Proof. Assume that $n=2$; by virtue of Definition 1 , we consider a family of $r_{2^{-}}$ rectangles, $R_{2, T}$, by choosing an arbitrary $T$ in the interval $\left(\frac{2 \pi k}{\log 2}, \frac{2 \pi(k+1)}{\log 2}\right)$ for each nonnegative integer $k$. Then, since $k<\frac{T \log 2}{2 \pi}<k+1$, we have $\left[\frac{T \log 2}{2 \pi}\right]=k$ 
and therefore, noticing (2.1), inside each $R_{2, T}$ there is exactly $k+1$ zeros of $L_{2}(z)$. Consequently

$$
N_{2}(T)=k+1
$$

and then the formula (1.5) is true for $n=2$.

Now we suppose that $n>2$ is a fixed integer. Noticing Definition 1 , we consider a generic $r_{n}$-rectangle $R_{n, T}$ and, as $L_{n}(z)$ is never 0 on its boundary, we are going to apply the argument principle. For this purpose, firstly, for a given $0<\epsilon<\frac{1}{6}$, by virtue of (2.2) and (2.3), we determine two values $a_{n}^{\prime \prime}$, $b_{n}^{\prime \prime}$, with $a_{n}^{\prime \prime}<a_{n}^{\prime}$ and $b_{n}^{\prime \prime}>b_{n}^{\prime}$, satisfying

$$
\left|L_{n}(z)-1\right|<\sin \frac{\epsilon}{6} \text { for all } z \text { with } \operatorname{Re} z=a_{n}^{\prime \prime}
$$

and

$$
\left|\frac{L_{n}(z)}{n^{z}}+1\right|<\sin \frac{\epsilon}{6} \text { for all } z \text { with } \operatorname{Re} z=b_{n}^{\prime \prime} .
$$

Secondly, we define a new $r_{n}$-rectangle, say $R_{n, T}^{\prime}$, bounded by the vertical lines $x=a_{n}^{\prime \prime}, x=b_{n}^{\prime \prime}$ and the paths

$$
\begin{gathered}
\Gamma_{n}^{\prime \prime}:=\left\{(x, y): a_{n}^{\prime \prime} \leq x \leq \alpha_{n}-r_{n} ; y=0\right\} \cup\left\{z:\left|z-\alpha_{n}\right|=r_{n} ; \operatorname{Im} z<0\right\} \cup \\
\cup\left\{(x, y): \alpha_{n}+r_{n} \leq x \leq b_{n}^{\prime \prime} ; y=0\right\}, \\
\Gamma_{n, T}^{\prime \prime}:=\left\{z+i T: z \in \Gamma_{n}^{\prime \prime}\right\},
\end{gathered}
$$

and according to $\operatorname{Re} z \leq a_{n}^{\prime}$ and $\operatorname{Re} z \geq b_{n}^{\prime}$ are zero-free regions, we conclude that $L_{n}(z)$ has the same number of zeros inside both $r$-rectangles $R_{n, T}$ and $R_{n, T}^{\prime}$. Then, for the previous $\epsilon$, we claim that there exist infinitely many values of $T$ such that the variation of the argument of $L_{n}(z)$ on the boundary of $R_{n, T}^{\prime}$, denoted by $V A\left(L_{n}(z) ; R_{n, T}^{\prime}\right)$, satisfies

$$
V A\left(L_{n}(z) ; R_{n, T}^{\prime}\right)=T \log n+2 \pi+\theta, \text { with }|\theta|<\epsilon .
$$

Indeed, from (2.6), the variation of the argument of $L_{n}(z)$ on the side of $R_{n, T}^{\prime}$ defined by the line $x=a_{n}^{\prime \prime}$, say $V A\left(L_{n}(z) ; x=a_{n}^{\prime \prime}\right)$, verifies

$$
\left|V A\left(L_{n}(z) ; x=a_{n}^{\prime \prime}\right)\right|<\frac{\epsilon}{3} .
$$

By writing

$$
L_{n}(z)=e^{z \log n} \frac{L_{n}(z)}{n^{z}}
$$

and taking into account (2.7), we conclude that the variation of the argument of $L_{n}(z)$ on the side of $R_{n, T}^{\prime}$ defined by the line $x=b_{n}^{\prime \prime}, V A\left(L_{n}(z) ; x=b_{n}^{\prime \prime}\right)$, is given by

$$
V A\left(L_{n}(z) ; x=b_{n}^{\prime \prime}\right)=T \log n+\alpha, \text { with }|\alpha|<\frac{\epsilon}{3}
$$

On the other hand, by noting that $\frac{\pi}{\log n}>r_{n}>0$, it follows that $\operatorname{Im} L_{n}(z)>0$ for $z$ satisfying $\left|z-\alpha_{n}\right|=r_{n}$ with $\operatorname{Im} z<0$. Furthermore, since $L_{n}(z)$ is one-to-one on $\left|z-\alpha_{n}\right| \leq r_{n}$, the curve defined by $L_{n}(z)$ when $z \in \Gamma_{n}^{\prime \prime}$ is:

i) the real segment from the point $L_{n}\left(a_{n}^{\prime \prime}\right)>0$ to $L_{n}\left(\alpha_{n}^{\prime \prime}-r_{n}\right)>0$,

ii) a path contained in the upper half-plane joining the point $L_{n}\left(\alpha_{n}^{\prime \prime}-r_{n}\right)$ with $L_{n}\left(\alpha_{n}^{\prime \prime}+r_{n}\right)<0$, and

iii) the real segment from the point $L_{n}\left(\alpha_{n}^{\prime \prime}+r_{n}\right)$ to $L_{n}\left(b_{n}^{\prime \prime}\right)<0$. 
Therefore, the variation of the argument of $L_{n}(z)$ when $z$ lies on $\Gamma_{n}^{\prime \prime}$, denoted by $V A\left(L_{n}(z) ; z \in \Gamma_{n}^{\prime \prime}\right)$, verifies

$$
V A\left(L_{n}(z) ; z \in \Gamma_{n}^{\prime \prime}\right)=\pi
$$

Finally, it only remains to prove that there exist infinitely many values of $T$ for which the variation of the argument of $L_{n}(z)$ on the path $\Gamma_{n, T}^{\prime \prime}$, denoted by $V A\left(L_{n}(z) ; z \in \Gamma_{n, T}^{\prime \prime}\right)$, satisfies

$$
V A\left(L_{n}(z) ; z \in \Gamma_{n, T}^{\prime \prime}\right)=\pi+\beta, \text { with }|\beta|<\frac{\epsilon}{3} .
$$

Indeed, let $\left\{p_{1}, p_{2}, \ldots, p_{k_{n}}\right\}$ be the set of all prime numbers less than or equal to $n$. Then, by expressing for each $m \in\{1,2, \ldots, n\}$

$$
\log m=\sum_{j=1}^{k_{n}} l_{m, j} \log p_{j}, l_{m, j} \text { nonnegative integers, }
$$

and substituting in $L_{n}(z)$, the functions $\operatorname{Re} L_{n}(z)$ and $\operatorname{Im} L_{n}(z)$ become polynomials in

$$
e^{x \ln p_{j}}, \cos \left(y \log p_{j}\right), \sin \left(y \log p_{j}\right), j=1, \ldots, k_{n} .
$$

Since $r_{n}$ has been chosen such that $L_{n}(z) \neq 0$ for all $0<\left|z-\alpha_{n}\right| \leq r_{n}$, we can assure that

$$
m_{n}:=\min \left\{\left|L_{n}(z)\right|:\left|z-\alpha_{n}\right|=r_{n} ; \operatorname{Im} z \leq 0\right\}>0
$$

and, consequently, we can select $\epsilon^{\prime}>0$ such that

$$
\epsilon^{\prime}<\min \left\{m_{n}, \min \left\{L\left(a_{n}^{\prime \prime}\right),-L\left(b_{n}^{\prime \prime}\right)\right\} \tan \frac{\epsilon}{6}\right\} .
$$

By continuity, there exists $\delta>0$ such that for any $z \in \Gamma_{n}^{\prime \prime}$ one has

$$
\left|L_{n}(z+i t)-L_{n}(z)\right|<\epsilon^{\prime}
$$

provided that

$$
\left|\cos \left(t \log p_{j}\right)-1\right|<\delta,\left|\sin \left(t \log p_{j}\right)\right|<\delta, \text { for all } j=1, \ldots, k_{n} .
$$

Then, given $\delta>0$, we determine a positive $\eta<\epsilon^{\prime}$ such that for any $\eta_{j}$ satisfying $\left|\eta_{j}\right| \leq \eta$, we have

$$
\left|\cos \left(\eta_{j} \log p_{j}\right)-1\right|<\delta,\left|\sin \left(\eta_{j} \log p_{j}\right)\right|<\delta, \text { for all } j=1, \ldots, k_{n} .
$$

Now we consider the numbers

$$
\alpha_{j}:=\frac{2 \pi}{\ln p_{j}}, \beta_{j}:=0,1 \leq j \leq k_{n} ; 1 ; \frac{\eta \log 2}{2 \pi},
$$

and since the $\frac{1}{\alpha_{j}}$ 's are linearly independent over the rationals, from Kronecker's theorem [3, Theorem 444], there exists $t_{1}>1$ and integers $q_{1, j}$ such that

$$
\left|t_{1} \frac{1}{\alpha_{j}}-q_{1, j}\right|<\frac{\eta \log 2}{2 \pi}, \text { for all } j=1, \ldots, k_{n} .
$$

Multiplying by $\alpha_{j}$ we have

$$
\left|t_{1}-\alpha_{j} q_{1, j}\right| \leq \eta, \text { for all } j=1, \ldots, k_{n} .
$$


Then by defining $\eta_{j}:=t_{1}-\alpha_{j} q_{1, j}$, we get $\left|\eta_{j}\right| \leq \eta$ and so (2.16) is fulfilled. Moreover, as $\cos \left(t_{1} \log p_{j}\right)=\cos \left(\eta_{j} \log p_{j}\right)$ and $\sin \left(t_{1} \log p_{j}\right)=\sin \left(\eta_{j} \log p_{j}\right)$ for all $j=1, \ldots, k_{n}$, inequality $(2.15)$ is verified for $t=t_{1}$ and then (2.14) follows. That is

$$
\left|L_{n}\left(z+i t_{1}\right)-L_{n}(z)\right|<\epsilon^{\prime}, \text { for all } z \in \Gamma_{n}^{\prime \prime},
$$

which means that the curve $L_{n}\left(z+i t_{1}\right)$ is contained in the $\epsilon^{\prime}$-neighborhood of the curve $L_{n}(z)$ when $z \in \Gamma_{n}^{\prime \prime}$. Hence, for $T=t_{1}$, from (2.11) and (2.13), the variation of the argument of $L_{n}(z)$ when $z \in \Gamma_{n, T}^{\prime \prime}$, denoted by $V A\left(L_{n}(z) ; z \in \Gamma_{n, T}^{\prime \prime}\right)$, satisfies

$$
V A\left(L_{n}(z) ; z \in \Gamma_{n, T}^{\prime \prime}\right)=\pi+\beta, \text { with }|\beta|<\frac{\epsilon}{3},
$$

which implies that (2.12) is true, as claimed. Now, taking into account (2.9), (2.10), (2.11) and (2.12), we obtain (2.8). Dividing that by $2 \pi$, the desired formula $(1.5)$ follows for the value $T=t_{1}$. Inductively, by considering the numbers

$$
\alpha_{j}:=\frac{2 \pi}{\ln p_{j}}, \beta_{j}:=0,1 \leq j \leq k_{n} ; t_{l} ; \frac{\eta \log 2}{2 \pi},
$$

from Kronecker's theorem, there exist $t_{l+1}>t_{l}$ and integers $q_{l+1, j}$ such that

$$
\left|t_{l+1} \frac{1}{\alpha_{j}}-q_{l+1, j}\right|<\frac{\eta \log 2}{2 \pi} \text {, for all } j=1, \ldots, k_{n} .
$$

Then repeating verbatim the above reasoning, the formula (1.5) is true for $T=t_{l+1}$. Therefore the sequence $\left(t_{1}, t_{2}, \ldots, t_{l}, \ldots\right)$ of values for $T$ validates $(1.5)$ and then the theorem follows.

\section{NUMERICAL EXPERIENCES}

As we have seen in the proof of our main result, for a given $n>2$, the existence of the values of $T$ for which the formula (1.5) is true is assured by Kronecker's theorem. In table 1, by diophantine approximation we have obtained some $T$ 's for different values of $n$ for which we have computed the exact number of zeros, $N_{n}(T)$, of $L_{n}(z)$ inside each $r_{n}$-rectangle $R_{n, T}$ and the corresponding value of $\frac{T \log n}{2 \pi}$. This allows to show the usefulness and the efficiency of (1.5).

\section{REFERENCES}

[1] Ash, R.B.: Complex Variables. Academic Press, New York (1971).

[2] Gonek, S.M., Ledoan, A.H.: Zeros of partial sums of the Riemann Zeta-function. Int. Math. Res. Not. no. 10, 1775-1791 (2010).

[3] Hardy, G.H., Wright, E.M.: An introduction to the Theory of Numbers. Clarendon Press, Oxford, UK (1954).

[4] Langer, R.E.: On the zeros of exponential sums and integrals. Bull. Amer. Math. Soc. 37, 213-239 (1931).

[5] Lapidus, M.L., Pomerance, C.: The Riemann zeta-function and the one-dimensional WeylBerry conjecture for fractal drums. Proc. London Math. Soc. 66 (3), 41-69 (1993).

[6] Lapidus, M.L., van Frankenhuijsen, M.: Fractal Geometry, Complex Dimensions and Zeta Functions. Springer-Verlag, New York (2006).

[7] Lapidus, M.L.: In search of the Riemann zeros. AMS, USA (2008).

[8] Levin, B.J.: Distribution of Zeros of Entire Functions. Amer. Math. Soc., Providence (1980).

[9] Pólya, G., JBer. Deutsch. Math.-Verein, 3, p. 97 (1925), Problem 24.

[10] Tamarkin, J.D.: The zeros of certain integral functions. J. London Math. Soc. 2, 66-69 (1927).

[11] Tamarkin, J.D.: Some general problems of the theory of ordinary linear differential equations and expansions of an arbitrary function in series of fundamental functions. Math. Z. 27, 1-54 (1928). 


\begin{tabular}{|c|c|c|c|}
\hline $\begin{array}{c}\text { Value } \\
\text { of n }\end{array}$ & $\begin{array}{c}\text { Value } \\
\text { of } T\end{array}$ & $\begin{array}{c}\text { Total number } \\
\text { of zeros in } R_{n, T}\end{array}$ & $\begin{array}{c}\text { Decimal Value of } \\
\frac{T \log n}{2 \pi}\end{array}$ \\
\hline 3 & 1000 & 175 & 174.8495763 \\
5 & 500 & 129 & 128.0749996 \\
5 & 1500 & 385 & 384.2249989 \\
6 & 1000 & 286 & 285.1673763 \\
7 & 1000 & 310 & 309.7012190 \\
10 & 500 & 184 & 183.2338997 \\
10 & 2500 & 917 & 916.1694985 \\
15 & 500 & 216 & 215.4997878 \\
25 & 750 & 385 & 384.2249989 \\
30 & 1000 & 542 & 541.3173755 \\
40 & 750 & 441 & 440.3275496 \\
53 & 2500 & 1580 & 1579.728959 \\
55 & 500 & 319 & 318.8934425 \\
\hline
\end{tabular}

TABlE 1. Numerical experiences

[12] Turán, P.: On some approximative Dirichlet-polynomials in the theory of the zeta-function of Riemann. Danske Vid. Selsk. Mat.-Fys. Medd., 24, no. 17, 1-36 (1948).

[13] Wilder, C.E.: Expansion problems of ordinary linear differential equations with auxiliary conditions at more than two points. Trans. Amer. Math. Soc. 18, 415-442 (1917).

Departamento de Análisis Matemático, Universidad de Alicante, 03080 Alicante, SPAIN

E-mail address: gaspar.mora@ua.es

Departamento de Análisis Matemático, Universidad de Alicante, 03080 Alicante, SPAIN

E-mail address: JM.Sepulcre@ua.es 